Urologe $2021 \cdot 60: 1468-1472$

https://doi.org/10.1007/s00120-021-01666-7

Online publiziert: 27. September 2021

○) Springer Medizin Verlag GmbH, ein Teil von

Springer Nature 2021

\section{Berufliche Trauer}

\section{Zum funktionalen Umgang mit Todesfällen in der Onkologie}

München, Deutschland

Trauer stellt eine natürliche Reaktion auf den Verlust von Personen oder Dingen dar, zu denen eine emotionale Bindung bestanden hat. In der Onkologie entstehen infolge langer Krankheitsverläufe und der für die Betroffenen existenziellen Situation mitunter Beziehungen, die von Nähe und Sympathie geprägt sind. Der Tod dieser Patientinnen und Patienten kann Elemente von Trauer hervorrufen.

Für entsprechende Trauerreaktionen hat sich im angloamerikanischen Sprachraum der Begriff „professional grief" etabliert, der im vorliegenden Beitrag mit beruflicher Trauer übersetzt wird. Private Trauer unterscheidet sich von beruflicher vor allem im Hinblick auf das Ausmaß des Einschnitts in bedeutsame Lebensbereiche und deren erforderliche Neuausrichtung. Demgegenüber sind in beruflicher Trauer spezifische Elemente enthalten, die in der privaten Trauer fehlen: Die Häufung von Todesfällen und die Mitverantwortung für den Krankheits- und Sterbeprozess. Über die private Trauer Hinterbliebener liegt eine Fülle theoretischen und empirischen Wissens vor. Demgegenüber existieren nur wenige systematische Untersuchungen zur beruflichen Trauer aufseiten der behandelnden Fachkräfte.

\section{Empirische Befunde zu beruflicher Trauer}

Zwölf Untersuchungen zu beruflicherTrauer von Onkologinnen und Onkologen wurden seit dem Jahr 2000 veröffentlicht. Die dafür Befragten stammen aus Kanada [2, $8,10]$, den USA [18, 22], Israel [6, 7, 9, 12], Spanien [19] und Griechenland [17]. Hinzu kommen theoretische Publikationen [1, $5,16,20]$. Weitaus häufiger wird berufliche
Trauer im Hospiz- und Palliativsetting sowie bei Pflegenden untersucht (für einen aktuellen Überblick vgl. [3]).

\section{Häufigkeit und Intensität}

Etwa $50 \%$ der Befragten äußern, dass sie sich von Todesfällen ihrer Patient*innen belastet fühlen. Dabei liegt die Belastungsschwere in mittleren Intensitätsbereichen: 4,7 auf einer 10er-Skala (Befragung von

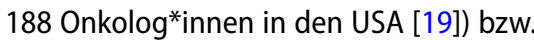
26 auf einer Summenskala von 13 bis 65 (71 Onkolog*innen in Israel $[11,12])$. In einer weiteren Befragung von 79 israelischen Onkolog*innen betrachten es zwar $80 \%$ als Gewinn, durch die Konfrontation mit dem Sterben einen vertieften Blick auf das Leben zu bekommen; allerdings leiden auch $70 \%$ unter emotionaler Erschöpfung und Burn-out- Symptomen infolge häufiger Todesfälle [6]. Diese Trauerfolgen beschränken sich nicht auf den Zeitpunkt des Todes; sie entwickeln sich bereits bei der Diagnose eines Progresses, beim Antizipieren absehbarer Todesfälle und beim Übermitteln schlechter Nachrichten [5, 9, 10].

\section{Charakteristika}

Nach den Elementen beruflicher Trauer gefragt, berichten Onkolog*innen von Traurigkeit, Leere, Mitgefühl mit Angehörigen, intrusiven Gedanken und Bildern bis hin zum Träumen von Verstorbenen, Aufgewühltsein, empfundener Machtlosigkeit, Selbstzweifeln und grundsätzlichen Gedanken über Endlichkeit, Schicksal und Sinnfragen [10, 17, 18]. Papadatou [16] schlägt eine Differenzierung von sechs Aspekten vor, die durch berufliche Todesfälle berührt werden können ( $\bullet$ Tab. 1): der Verlust von zum Teil besonderen Beziehungen, das Mitfühlen mit dem Schmerz 


\begin{tabular}{|l|l|}
\hline \multicolumn{2}{|l|}{$\begin{array}{l}\text { Tab. } 1 \text { Charakteristika beruflicher Trau- } \\
\text { er. (Nach Papadatou [16]) }\end{array}$} \\
\hline 1. & Verlust einer besonderen Beziehung \\
\hline 2. & $\begin{array}{l}\text { Mitfühlen mit dem Schmerz Hinter- } \\
\text { bliebener }\end{array}$ \\
\hline 3. & $\begin{array}{l}\text { Selbstzweifel, Machtlosigkeit, Schuld- } \\
\text { gefühle }\end{array}$ \\
\hline 4. & $\begin{array}{l}\text { Infragestellen eigener Grundüberzeu- } \\
\text { gungen }\end{array}$ \\
\hline 5. & $\begin{array}{l}\text { Erinnerung an zurückliegende sowie } \\
\text { antizipierte Verluste im eigenen Leben }\end{array}$ \\
\hline 6. & $\begin{array}{l}\text { Konfrontation mit der eigenen Sterb- } \\
\text { lichkeit }\end{array}$ \\
\hline
\end{tabular}

Hinterbliebener, besonders von Kindern, Selbstzweifel hinsichtlich des eigenen therapeutischen Handelns, die Erschütterung von Grundüberzeugungen hinsichtlich eines kontrollierbaren Lebens und die Konfrontation mit der eigenen Verlustbiografie und der eigenen Sterblichkeit.

\section{Verlauf}

In der Trauerpsychologie hat bereits in den 1990er-Jahren eine Abkehr von den früher populären Phasenmodellen stattgefunden, weil diese empirisch widerlegt waren und die Gefahr einer Normierung von Trauerverläufen bargen.

\section{I) In der Trauerpsychologie wurden die früheren Phasenmodelle vom dualen Prozessmodell abgelöst}

Sie wurden vom sog. dualen Prozessmodell [21] abgelöst, das auch für berufliche Trauerverläufe gilt [16] und diese als natürliche Fluktuation zwischen zwei psychischen Prozessen beschreibt: Der verlustorientierte Prozess beinhaltet das Wahrnehmen und Ausdrücken von Trauerreaktionen; der wiederherstellungsorientierte Prozess umfasst das Vermeiden und Unterdrücken von Trauer zugunsten eines Funktionierens im Alltag und einer Konzentration auf das eigene Leben. Beide Prozesse gelten als normal und adaptiv, da erst ihr Zusammenspiel ermöglicht, eine Verlusterfahrung zu betrauern, ohne von ihr überwältigt zu werden.

Tab. 2 Handlungsmöglichkeiten für den Umgang mit beruflicher Trauer in drei Handlungsfeldern

\begin{tabular}{|c|c|}
\hline $\begin{array}{l}\text { Handlungs- } \\
\text { feld }\end{array}$ & Handlungsmöglichkeit \\
\hline \multirow{3}{*}{$\begin{array}{l}\text { Behandelte } \\
\text { und Ange- } \\
\text { hörige } \\
\text { (perimortal) }\end{array}$} & Die eigene Position zwischen Empathie und Abgrenzung finden \\
\hline & $\begin{array}{l}\text { Kontaktaufnahmemit Hinterbliebenen: Anruf, Kondolenzkarte, Einladung zum } \\
\text { Gespräch oder Teilnahme an Bestattungsritualen }\end{array}$ \\
\hline & Inneres Verabschieden: persönliche Rituale \\
\hline \multirow{5}{*}{$\begin{array}{l}\text { Die eigene } \\
\text { Person }\end{array}$} & Die eigene Reaktion wohlwollend akzeptieren \\
\hline & $\begin{array}{l}\text { BewusstesWegschieben trauerbezogener Gedanken und Gefühle („compartmen- } \\
\text { talization“) }\end{array}$ \\
\hline & Bewusste Lebensgestaltung, Spiritualität und Selbstfürsorge \\
\hline & Palliative Versorgung als Teilbereich der eigenen Arbeit definieren \\
\hline & Aussprache im beruflichen oder privaten Umfeld (informell oder formell) \\
\hline \multirow[t]{3}{*}{ Das Team } & (Mit-)Teilen von Emotionen \\
\hline & Anerkennen und Normalisieren von Trauer \\
\hline & Gedenkrituale im Team \\
\hline
\end{tabular}

\section{Handlungsmöglichkeiten bei beruflicher Trauer}

In den meisten Selbstbeschreibungen von ärztlichem und pflegendem Fachpersonal in der Onkologie findet sich eine Kombination der beiden Prozesse Hinwendung zur Trauer und Hinwendung zum Leben $[8,17]$. Da jede Person über eigene Lebenserfahrungen, Persönlichkeitseigenschaften und Glaubensvorstellungen verfügt, folgt auch der Umgang mit beruflichen Verlusterfahrungen individuellen Handlungslinien. Entsprechend zeigt sich empirisch eine große Heterogenität in den von Onkolog*innen berichteten Coping-Strategien [8], sodass sich keine Pauschalempfehlungen für den Umgang mit beruflicher Trauer formulieren lassen und die nachfolgenden Ausführungen individuell zu gewichtende Anregungen darstellen.

\section{》) Empirisch zeigt sich eine große Heterogenität in den berichteten Coping-Strategien}

- Tab. 2 gibt einen Überblick über CopingStrategien, die von Onkologinnen und Onkologen in verschiedenen Befragungen genannt werden $[8,10,18]$.
Handlungsfeld 1: Die Beziehung zum perimortalen Menschen

Willst du, dass etwas klein wird, mache es groß. (Chinesisches Sprichwort)

Die eigene Position zwischen Empathie und Abgrenzung finden Empathie mit Patient*innen ist differenziert zu betrachten: Sie korreliert bei vielen Ärzt* innen mit Arbeitszufriedenheit. Andererseits kann ein starkes Mitfühlen ohne die Fähigkeit zur anschließenden Distanzierung berufliche Trauer unangemessen verstärken [11]. In einer Untersuchung kanadischer Onkolog*innen versuchte die Hälfte der Befragten, ihre Trauerreaktion abzumildern, indem sie weniger Energie auf die Versorgung terminaler Patient*innen verwendete und seltener Termine oder Visiten durchführte [10]. Davon abgesehen, dass Menschen in Todesnähe ärztliche Zuwendung und empathische Kommunikation über Behandlungswünsche benötigen, kann der Eindruck, sich aus Selbstschutz distanziert zuhaben, bei Behandelnden Schuldgefühle auslösen und die berufliche Trauer verkomplizieren $[8,9]$. Deshalb kann es durchaus im eigenen Interesse liegen, den Kontakt in Todesnähe so zu gestalten, dass möglichst wenig Versäumtes zurückbleibt. Ziel ist dann nicht mehr professionelle Distanz, sondern „professionelle Nähe" [14]. 
Kontaktaufnahme mit Hinterbliebenen

Eine Kontaktaufnahme mit Hinterbliebenen erfolgt meist per Telefon, Kondolenzkarte oder Einladung zu einem Nachgespräch. Von 535 befragten kanadischen Onkolog*innen nutzten $21 \%$ diese Möglichkeiten regelhaft, ein weiteres Drittel manchmal, wobei am häufigsten das Medium des Telefonats genutzt wurde, gefolgt von der Kondolenzkarte [2]. In der Palliativmedizin gilt das Nachgespräch mit Hinterbliebenen als Qualitätsmerkmal einer guten Palliativversorgung und wird entsprechend häufiger eingesetzt (ebd.). Als häufigste Hürden werden von Onkolog*innen Zeitmangel (40\%) und Unsicherheit im Umgang mit trauernden Angehörigen (14\%) genannt [2]. Im Hinblick auf die berufliche Trauer ist die Kontaktaufnahme insofern relevant, als sie nicht nur den Hinterbliebenen Trost spendet, sondern auch den eigenen inneren Abschluss erleichtert.

\section{Verstorbene innerlich} verabschieden, persönliche Rituale Persönliche Abschiedsrituale beinhalten beispielsweise Kondolenzanruf oder Trauerkarte, eine kurze Reflexion des gemeinsam gegangenen Wegstücks, das Anzünden einer Kerze, einen Spaziergang, ein Gebet oder das Imaginieren der verstorbenen Person mit dem Wunsch einer "guten Reise" [15]. Als Gegengewicht zum Nichtsmehr-tun-Können schaffen Rituale einen Rückgewinn an Handlungsmöglichkeiten. Abschiedsrituale nach dem Tod haben in fast allen Kulturen einen Bezug zum Danach. Dieser kann einen religiösen Kern implizieren (etwa im christlichen Sterbegebet "Zum Paradies mögen Engel dich geleiten"), benötigt diesen aber nicht zwingend: Auch aus atheistischer Perspektive ist es möglich, einen Verstorbenen imaginativ dem „Nichts“ zu überantworten. Abschiedsrituale korrelieren im Hospizsetting mit Empathie und Arbeitszufriedenheit als Indikatoren für gut bewältigte berufliche Trauer [15].

\section{Handlungsfeld 2: Der Umgang mit sich selbst}

Jeder, der geht, belehrt uns ein wenig über uns selber. Kostbarster Unterricht an den Sterbebetten. (Hilde Domin)
Akzeptieren der eigenen Reaktion Wie vom dualen Prozessmodell konzipiert, fluktuiert die Trauerreaktion zwischen dem Erleben und dem Vermeiden verlustbezogener Gedanken und Gefühle. So schwanken wir zuweilen zwischen Traurigkeit, Leere, Nachdenklichkeit einerseits und Gleichgültigkeit oder dem Bedürfnis nach Ablenkung andererseits. Von der eigenen, manchmal widersprüchlichen Reaktion nicht irritiert zu werden, sondern sie wohlwollend zu akzeptieren, wird sowohl in der allgemeinen Trauerpsychologie wie auch in den Arbeiten zur beruflichen Trauer als wichtiger Faktor eines gesunden Trauerverlaufs betont [17]. Giddings [5] formuliert als Onkologe das Ziel ,... to honor my grief, whatever form it may take, as grief allowsme to honor the many patients..." (S. 1357).

\section{Bewusstes Wegschieben \\ („,compartmentalization“)}

Unterdrücken, Ablenken und Verdrängen stehen oft im Verdacht, dysfunktional zu sein. Tatsächlich kann aber das bewusste Beiseitestellen, das im Englischen als ", compartmentalization" bezeichnet wird, eine funktionale Strategie darstellen, sofern sie nicht die einzige bleibt. Wer ausschließlich auf diese Strategie festgelegt ist, wird emotional abstumpfen. Im Kontext eines flexiblen Verhaltensrepertoires stellt das Unterdrücken von Gefühlen jedoch eine Handlungsoption dar, die es uns ermöglicht, weiter zu funktionieren und uns beispielsweise auf das nächste Behandlungsgespräch zu konzentrieren. In Befragungen geben nahezu alle Onkolog*innen an, dass sie diese Strategie anwenden, indem sie sich auf anderes konzentrieren und ihre Gefühle bewusst unterdrücken $[7,8,17,18]$.

\section{Bewusste Lebensgestaltung,} Spiritualität und Selbstfürsorge

Berufliche Todesfälle konfrontieren uns mit der Kostbarkeit auch unseres eigenen Lebens. Diese Erinnerung an die eigene Sterblichkeit dient vielen Befragten als Ansporn, bewusst in der Gegenwart zu leben, enge Beziehungen in der Familie und zu Freunden zu pflegen, Interessen und Hobbies zu verfolgen, auf Sport, Erholung und Schlaf zu achten und sich je nach persönlicher Bedeutung Lebensphilosophien, Glaubensvorstellungen und Spiritualität zuzuwenden [8]. Dieses Maßnahmenbündel, das individuell gewichtet auf das eigene biopsychosoziale Wohlbefinden abzielt, wird als Selbstfürsorge bezeichnet. Das Praktizieren einer guten Selbstfürsorge sagte in einer Befragung von 387 spanischen Palliativmediziner* innen am besten vorher, wie gut diese mit beruflicher Trauer zurechtkamen [19].

Palliative Versorgung als Teilbereich der eigenen Arbeit definieren Obwohl Palliativmediziner*innen häufig mit Todesfällen konfrontiert sind, leiden sie nicht stärker unter trauerbezogenen Belastungen als andere fachärztliche Gruppen [13]. Granek et al. [6] führen dies auf die spezifische Zielsetzung der palliativmedizinischen Arbeit zurück: Durch die Fokussierung der Interventionen auf die supportive Begleitung von Betroffenen und ihren Familien verändert sich der an das eigene berufliche Handeln angelegte Erfolgsmaßstab, sodass sich trotz des Todes häufig eine Zufriedenheit mit dem eigenen Beitrag zu einem würdevollen Sterbeprozess einstellt. Entsprechend litten Onkolog* innen in den USA, die sich ausdrücklich auch für die palliative Versorgung zuständig sahen, weniger unter beruflicher Trauer [18].

Entlastung durch Aussprache im beruflichen oder privaten Umfeld Die Hälfte bis zwei Drittel der Befragten gibt an, sich bei beruflicher Trauer im Gespräch mit anderen zu entlasten, ob im kurzen „Zwischen-Tür-und-Angel-Gespräch" oder im ausführlichen Austausch [8, 18]. Die verstorbene Person wird dadurch noch einmal kurz gewürdigt und es erfolgt eine emotionale Entlastung; beides erleichtert den inneren Abschluss. Allerdings merken Onkolog* innen an, dass es im Beruf häufig an geeigneten Gesprächspartner*innen fehlt und dass auch der Aussprache im privaten Umfeld Grenzen der Zumutbarkeit gesetzt sind [7].

Neben informellen Ad-hoc-Gesprächen kann eine Aussprache auch formalisiert in Supervision (einzeln oder in Gruppen), Mentoring-Programmen, Balint-Gruppen oder Debriefings erfolgen. Diesen Formaten kommt neben emotionaler Entlastung auch eine Fortbildungsfunktion im Hinblick auf das Selbstmanagement von Trauer zu [20]. 


\section{Handlungsfeld 3: Das Team}

Lange saßen sie dort und hatten es schwer. Aber sie hatten es gemeinsam schwer, und das war ein Trost. Leicht war es trotzdem nicht. (Astrid Lindgren:Ronja Räubertochter)

\section{(Mit-)Teilen von Emotionen}

Das oben besprochene Bedürfnis nach kollegialem Austausch macht berufliche Trauer zu einer Teamangelegenheit. Wie bereits erwähnt, kann das (Mit-)Teilen von Gefühlen emotional entlasten. Mit dem eigenen Erleben nicht allein zu sein, stärkt darüber hinaus die Kollegialität und die impliziten Werte eines Teams wie Verbindlichkeit, Ernsthaftigkeit und Solidarität [20].

Anerkennen beruflicher Trauer: Eine Aufgabe für Arbeitgeber und Leitende

Dass Einzelpersonen und Teams ihre Trauer ausdrücken, setzt innerhalb der Institution voraus, dass persönliche Betroffenheit nicht pauschal abgewertet wird. Häufig existieren im Team unausgesprochene Regeln darüber, worin eine adäquate professionelle Reaktion auf berufliche Todesfälle besteht. Diese Teamkultur in Richtung einer Offenheit für und Normalisierung von Gefühlen mitzuprägen, ist Aufgabe jedes Teammitglieds und insbesondere von Personen der Leitungsebene $[1,10,18]$. Insbesondere für Pflegende und medizinische Fachangestellte hat sich gezeigt, dass ihre Arbeitsqualität nach Todesfällen umso höher ist, je mehr ihre Trauerreaktion von ihren Vorgesetzten wertgeschätzt und legitimiert wird $[4,15]$.

\section{Rituale auf Teamebene}

Gegenüber individuellen Ritualen (vgl. Abschnitt "Verstorbene innerlich verabschieden, persönliche Rituale") bieten im Team praktizierte Rituale jene Vorteile, die sich aus dem Teilen von Emotionen ergeben: Die Solidarität, das implizite „Wir sind gemeinsam betroffen" bietet vielen Teammitgliedern Trost und schafft Verbundenheit. Durch Gedenkrituale wird zudem die Teamkultur dahingehend geprägt, dass berufliche Trauer als selbstverständlicher Teil der Arbeit anerkannt wird.
Praxisbeispiel:Das,,Rosen-Essen“1 Einmal jährlich versammeln sich alle Mitglieder einer onkologischen Praxis nach Feierabend am Flussufer. Dort verlesen sie die Namen der im zurückliegenden Jahr Verstorbenen und übergeben dem Wasser für jede Person eine Rose. Den Abend beschließen sie mit einem gemeinsamen Essen.

\section{Schlussbemerkung}

Berufliche Trauer ist keine pathologische Reaktion, die einer Intervention bedarf. Vielmehr sind Gefühle aufseiten der behandelnden Personen normal und angemessen, weshalb in diesem Beitrag für einen offenen und flexiblen Umgang mit beruflicher Trauer geworben wird.

\section{I) Berufliche Trauer ist keine pathologische Reaktion}

Für viele Onkolog*innen ist nicht die Trauer an sich belastend, sondern das Fehlen von Akzeptanz und Handlungsoptionen $[1,5]$. Eine weitere Hürde besteht im empfundenen Zeitmangel. Im übervollen Arbeitsalltag besteht wenig Raum für ein Innehalten, weder allein noch im Team. Dennoch gilt auch bei Zeitknappheit das scheinbare Paradoxon, dass selbst hohe Todeszahlen dann erträglich bleiben, wenn die eigene Trauer wahrgenommen und ausgedrückt werden kann.

\footnotetext{
1 Der Autor dankt Frau Agnes Blöchinger und Herrn Dr. med. Robert Eckert (onkologische Schwerpunktpraxis Esslingen) für die Schilderung des Rituals und die Erlaubnis zur Veröffentlichung.
}

Fazit für die Praxis

- Den Kontakt zu terminal Kranken und Hinterbliebenen so gestalten, dass möglichst wenig Versäumtes zurückbleibt („,professionelle Nähe")

- Ein persönliches Abschiedsritual entwickeln, das bei Bedarf als Handlungsoption zur Verfügung steht

- Trauer sowohl erleben als auch vermeiden können und das eigene Oszillieren zwischen beiden Prozessen zulassen

- Selbstfürsorge als Gegengewicht zur beruflichen Belastung praktizieren

- Beruflicher Trauer im Team einen adäquaten Platz einräumen

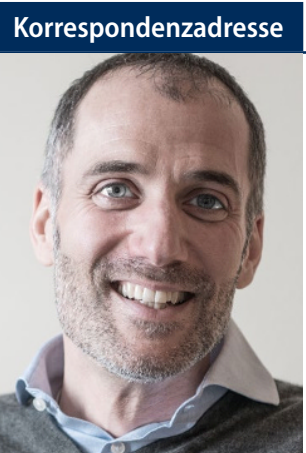

Dr. phil. Klaus Lang

Sendlinger-Tor-Platz 11, 80336 München, Deutschland mail@klauslang-online.de

Danksagung. Der Autor dankt Frau Dr. med. Agnes Huber und Herrn Dr. med. Carsten Oetzel für wertvolle Anregungen beim Erstellen des Manuskripts.

Einhaltung ethischer Richtlinien

Interessenkonflikt. K. Lang gibt an, dass kein Interessenkonflikt besteht.

Für diesen Beitrag wurden vom Autor keine Studien an Menschen oder Tieren durchgeführt. Für die aufgeführten Studien gelten die jeweils dort angegebenen ethischen Richtlinien. 


\section{Literatur}

1. Athanasios A (2020) Do doctors grieve: addressing physician grief and cultivating better doctors. Pastoral Psychol 69:83-85

2. Chau NG, Zimmermann C, Ma C et al (2009) Bereavement practices of physicians in oncology and palliative care. Arch Intern Med 169:963-971

3. Dijxhoorn AFQ, Brom L, van der Linden YM et al (2020) Prevalence of burnout in healthcare professionals providing palliative care and the effect of interventions to reduce symptoms: a systematic literature review. J Palliat Med. https:// doi.org/10.1177/0269216320956825

4. Engler-Gross A, Goldzweig G, Hasson-Ohayon l et al (2020) Grief over patients, compassion fatigue, and the role of social acknowledgment among psycho-oncologists. Psychooncology 29:493-499

5. Giddings $G$ (2010) The ties that bind: a reflection on physician grief. Support Care Cancer 18:1355-1357

6. Granek L, Ariad S, Nakash 0 et al (2017) Mixedmethods study of the impact of chronic patient death on oncologists' personal and professional lives. J Oncol Pract 13:e1-e10

7. Granek L, Ariad S, Shapira S et al (2016) Barriers and facilitators in coping with patient death in clinical oncology. Support Care Cancer 24:4219-4227

8. Granek L, Barbera L, Nakash 0 et al (2017) Experiences of Canadian oncologists with difficult patient deaths and coping strategies used. Curr Oncol Rep 24:e277
9. Granek L, Ben-David M, Shapira S et al (2017) Grief symptoms and difficult patient loss for oncologists in response to patient death. Psychooncology 26:960-966

10. Granek L, Tozer R, Mazzotta P et al (2012) Nature and impact of grief over patient loss on oncologists' personal and professional lives. Arch Intern Med 172:964-966

11. Hayuni G, Hasson-Ohayon I, Goldzweig G et al (2019) Between empathy and grief: the mediating effect of compassion fatigue among oncologists. Psychooncology 28:2344-2350

12. Laor-Maayany R, Goldzweig G, Hasson-Ohayon let al (2020) Compassion fatigue among oncologists: the role of grief, sense of failure, and exposure to suffering and death. Support Care Cancer 28:2025-2031

13. Pereira MS, Fonseca AM, Carvalho AS (2011) Burnout in palliative care: a systematic review. Nurs Ethics 18:317-326

14. Melching $H$ (2012) Zwischen dem Sterbe-Leben und dem Lebe-Leben. Persönliche Einschätzungen zum Schutzfaktor Privatleben. In: Müller M, Pfister D (Hrsg) Wieviel Tod verträgt das Team? Belastungs- und Schutzfaktoren in Hospizarbeit und Palliativmedizin. Vandenhoeck \& Ruprecht, Göttingen, S 238-249

15. Montross-Thomas LP, Scheiber C, Meier EA et al (2016) Personally meaningful rituals: a way to increase compassion and decrease burnout among hospice staff and volunteers. J Palliat Med 19:1043-1050
16. Papadatou D (2000) A proposed model of health professionals' grieving process. J Death Dying 41:59-77

17. Papadatou D, Bellali T, Papazoglou I et al (2002) Greek nurse and physician grief as a result of caring for children dying of cancer. Pediatr Nurs 28:345-354

18. Redinbaugh EM, Sullivan AM, Block SD et al (2003) Doctors' emotional reactions to recent death of a patient: cross sectional study of hospital doctors. BMJ 327:185-189

19. Sansó N, Galiana L, Oliver A et al (2015) Palliative care professionals' inner life: exploring the relationships among awareness, self-care, and compassion satisfaction and fatigue, burnout, and coping with death. J Pain Symptom Manage 50:200-207

20. Shanafelt T, Adjei A, Meyskens FL (2003) When your favorite patient relapses: physician grief and well-being in the practice of oncology. J Clin Oncol 21:616-2619

21. Stroebe M, Schut $H$ (1999) The dual process model of coping with bereavement: rationale and description. Death Stud 23:197-224

22. Whitehead PR (2014) The lived experience of physicians dealing with patient death. BMJ Support Palliat Care 4:271-276

\section{CME-Punkte sammeln in 3 Schritten}

Als Zeitschriftenabonnent stehen Ihnen unter www.SpringerMedizin.de/cme alle zertifizierten Fortbildungskurse Ihrer Zeitschrift zur Verfügung.

So einfach sammeln Sie CME-Punkte:

Registrieren

Um CME-Fortbildungen bearbeiten zu können, müssen Sie sich einmalig unter www.springermedizin.de/register registrieren. Bitte geben Sie bei der Registrierung die Lieferadresse Ihrer abonnierten Zeitschrift an, damit die Angaben Ihres OnlineAccounts mit den Angaben Ihres Zeitschriften-Abonnements übereinstimmen. Die CME-Beiträge werden anschließend automatisch freigeschaltet.

Beitrag auswählen

Sobald Sie sich mit Ihren Zugangsdaten angemeldet haben, können Sie auf www.SpringerMedizin.de/cme die gewünschten CME-Kurse Ihrer Zeitschrift nutzen. Die Kurse können jederzeit unterbrochen und später fortgesetzt werden.
CME-Punkte sammeln

Zu jedem Fortbildungskurs gehört ein Fragebogen mit 10 CMEFragen. Mit 7 richtigen Antworten haben Sie bestanden und erhalten umgehend eine Teilnahmebescheinigung!

Teilnehmen und weitere Informationen unter: www.SpringerMedizin.de/cme

Unser Tipp: Noch mehr Fortbildung bieten die e.Med-KombiAbos. Hier stehen Ihnen die CME-Kurse der Fachzeitschriften von Springer Medizin in elektronischer Form zur Verfügung. Auf Wunsch erhalten sie mit den e.Med-Kombi-Abos außerdem eine gedruckte Fachzeitschrift Ihrer Wahl.

Testen Sie e.Med kostenlos und unverbindlich!

Jetzt informieren unter

www.springermedizin.de $\Rightarrow$ "Abo-Shop" oder telefonisch unter 0800-77 80777

(Montag bis Freitag, 10 bis $17 \mathrm{Uhr}$ ) 